\title{
Zigbee as a Means to Reduce the Number of Blind Spot Incidents of a Truck
}

\author{
Steven De Lausnay, Thomas Standaert and Nobby Stevens \\ Catholic University College Ghent \\ Dept. of Engineering, DraMCo research Group \\ Gebroeders Desmetstraat 1 \\ B-9000 Gent, Belguim \\ E-mail: steven.delausnay@kahosl.be and \\ nobby.stevens@kahosl.be
}

\begin{abstract}
Every year in Europe, about 1500 people die in traffic because they are not noticed by the truck driver. This problem could be solved by developing a wireless communication system where the truck driver and the cyclist are informed about each others presence. In this paper a solution is presented in which the position of the cyclist is determined and displayed on a screen when being in the neighborhood of a truck. The cyclist gets an indication about notification by the truck. Because of the fast changing network, the cyclist must be added quickly to the network and the position must be updated very fast. For this reason a Zigbee communication system is used. The position of the cyclist is displayed in zones around the truck. The setup is experimentally tested and it demonstrated that the proposed solution leads to a reliable and fast method to reduce the number of blind spot incidents.
\end{abstract}

\section{INTRODUCTION}

Every year, people are injured and killed because of blind spot accidents. A contributing factor for this problem is that the truck driver does not notice them due to the fact that the current solutions for blind spot incidents require a lot of human intervention. There are three main solutions on the market. Firstly, there are those who make the blind spot visible. The most commonly used solution, is the blind spot mirror [1]. For this solution, the initiative of the truck driver to use the mirror correctly is necessary. Another solution for making the blind spot visible, is the placement of cameras [2]. Secondly, there are systems where the blind spot is marked on the truck. This way the cyclist gets an indication of the dangerous zones, but the truck driver is not informed of the presence of the cyclist. Examples of this solution are Blicor [3] and Life Saver [4]. Finally, there is a system that uses a sensor technique to detect the cyclist 's presence. The detection range of this system, called Lexguard [5], is approximately $1 \mathrm{~m}$. This way, the cyclist is only noticed when being in a dangerous situation. For all three solutions only one of the two participants are informed of each others presence.

The system in this paper, offers a solution where both participants are informed, also when the cyclist is situated in a safe position. Therefore some requirements are defined
Wout Joseph, member IEEE, Leen Verloock, Francis Goeminne and Luc Martens, member IEEE Ghent University/IBBT INTEC-WICA

Dept. of Information Technology

Gaston Crommenlaan 8 box 201

B-9050 Gent, Belguim

E-mail: wout.joseph@intec.UGent.be

TABLE I

REQUIREMENTS FOR THE SYSTEM

\begin{tabular}{ll}
\hline Criteria & Parameters \\
\hline Exactitude & $<5 \mathrm{~m}$ \\
Battery life & 3 years \\
Time to be noticed & $<1 \mathrm{~s}$ \\
Probability not noticed & $<0.01 \%$ \\
Cyclists in the network & $>20$ \\
Dimensions & $<100 \mathrm{~cm}^{3}$ \\
Minimal range & $>50 \mathrm{~m}$ \\
\hline
\end{tabular}

to select a suitable wireless technology. This technology is then used to develop a test setup to proof that a wireless technology can solve the blind spot problem. The accuracy of the system and the error of not noticing a cyclist, are evaluated with some experiments. Finally a conclusion and future work of this solution are given.

\section{REQUIREMENTS AND SELECTION OF A WIRELESS TECHNOLOGY}

Before considering the different possible technologies, it is important to define the requirements of the system. These are summarized in Table I.

Regarding these requirements a research was started on the available communication systems on the market. This has lead to six available wireless communication systems, namely Wifi, Bluetooth, GSM, UWB, GPS and Zigbee. $\mathrm{WiFi}$, Bluetooth and GSM were not an option. With WiFi the position error is too large and the battery life is too short. With Bluetooth, the connection time and positioning time are too large [6]. The same problem is found with GSM. Moreover the extra cost for the operator rights and agreements is an additional disadvantage [7]. GPS might be a solution but good GPS receivers are very expensive and use much energy. Beside GPS, a second communication system is required to make the connection between the truck and the 
cyclist [8]. For UWB, there were no commercial development tools on the market when the research took place so it was not possible to quickly build a test setup, but in the future the Sensor from Decawave [9] could be a solution.

For this reason a test setup is build using the Zigbee technology [10]. Zigbee is robust and has a low power consumption. The only disadvantage is the inaccuracy of the positioning [11]. To deal with this disadvantage, the solution makes use of zones.

\section{ZIGBEE AS A SOLUTION}

\section{A. General}

For the Zigbee test setup, the CC2430/31 from Texas Instruments [12] is used, because the $\mathrm{CC} 2431$ has a hardware location engine. The accuracy of the location engine is approximately $3 \mathrm{~m}$ [11], the update speed of the position is $600 \mathrm{~ms}$ with a lot of calculation time before using the hardware location engine. The program of the location engine is used as a starting point, but the position of the blind node is placed in zones instead of determining it exactly. This has the advantage that the truck driver can see the cyclists on the screen at once.

Four zones are defined in the immediate neighborhood of the truck. Every zone is represented by a reference node (Figure 1), this will bring four references nodes for zone 1 to zone 4 . In the cabin of the truck a location dongle is placed. This node will collect the information from the blind node (the cyclist) and display it on a screen. The location dongle will start up as coordinator of the network or if there is already a coordinator, as router. The reference nodes are always implemented as routers. The bike is equipped with a blind node that is configured as end device. This way it has a low power consumption.

For displaying the zones on the truck, a computer is used for testing purposes. In practice, this program can be implemented in the board computer of the truck or a hardware module can be developed and placed on the dashboard. The nodes in the truck will not be active at all times. This way the network is kept as small as possible so the attention goes out to the participants (trucks and cyclists) really needing it.

\section{B. Starting up the nodes at the truck}

The nodes on the truck are only active when the speed is below a vital level. Because the reference nodes need to know to which location dongle they belong, a startup routine is developed at these nodes. The startup routine also makes it possible that a truck can consist of different participants, the reference nodes are not bounded to one location dongle. As an example the configuration of Figure 1 shows two trucks determining the position of different cyclists.

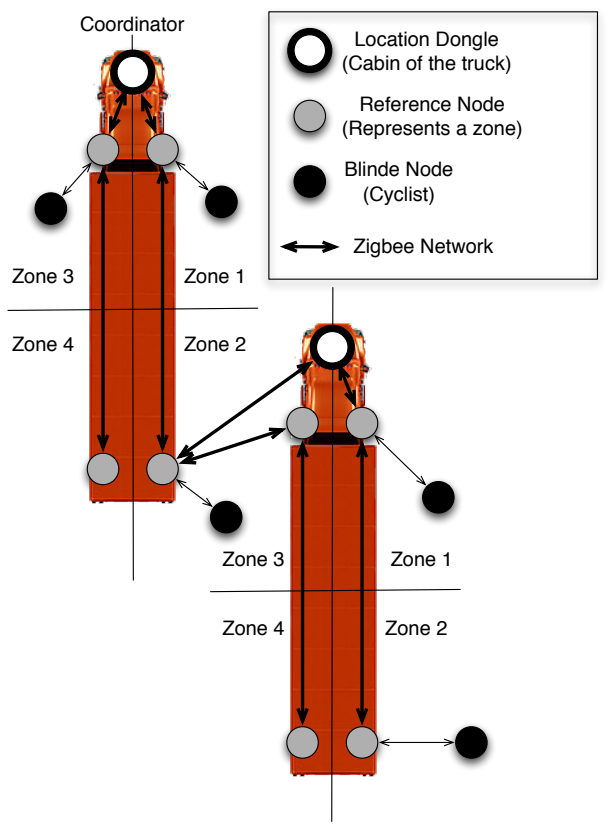

Fig. 1. Configuration with two trucks and different cyclists

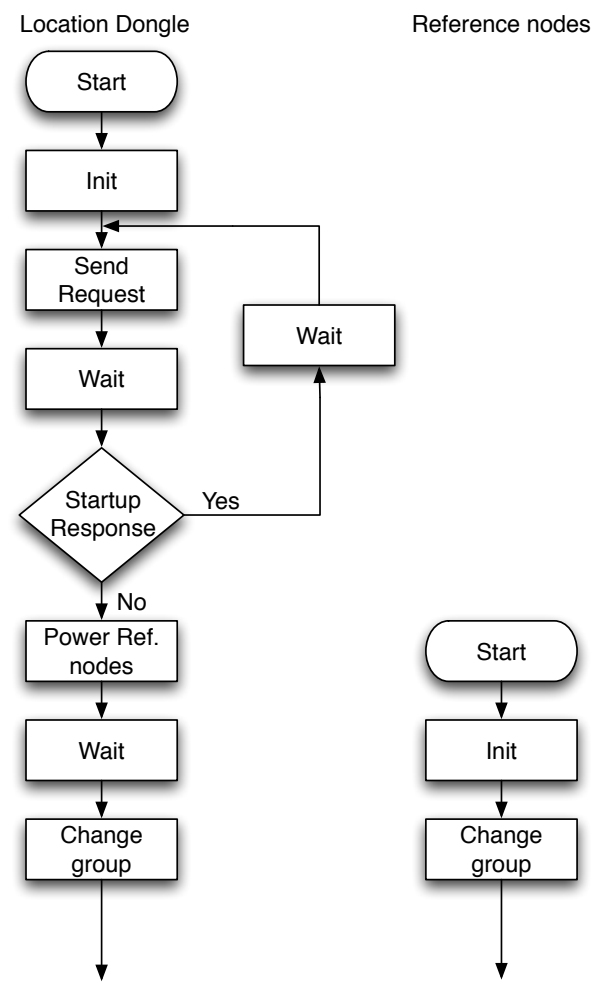

Fig. 2. The flowchart of the startup routines

Figure 2 shows a flowchart of the startup routine. The location dongle will start up when the speed is lower then a certain level that will be determined in a practical test. It performs board initializations and sends a message "Startup Request" to other location dongles. When the other location 
dongles in the network are also starting up, they will send an answer "Startup Response" to the location dongle making the request. The location dongle will wait for $500 \mathrm{~ms}$ and then will try again. If there are no answers in $500 \mathrm{~ms}$, the location dongle will activate the connected reference nodes. The location dongle then waits for a certain amount of time (currently set to $500 \mathrm{~ms}$ ) to enable the reference nodes to perform their initializations. Once this is done the reference nodes are set in group zero. The location dongle sends a message to group zero with a new group number. The new group number will be the location dongle short address plus one. Now the references nodes know to which location dongle they belong. The startup is completed for the location dongle and reference nodes and they can start to determine the potential presence of cyclists. With this startup routine only two wires are needed to connect all nodes namely the power supply of the nodes.

By using this startup routine it is also possible to determine if there is a reference node that did not start up. After the group number has changed, the reference nodes could send a message to the location dongle that they are activated and represent a zone. If a reference node does not respond to the group change, this can be communicated to the truck driver and the zone is not loaded on the screen.

\section{Zone determination}

For the zone determination we investigate the data messages between the different nodes in the network (Figure 3). When the cyclist approaches a moving truck, it will make connection and join the network. The blind node then starts with a blast. A blast is a series of messages that are not routed through the network and with no information. The reference nodes use the blast to get the Link Quality Indicator (LQI) from the messages. The reference nodes save the LQI in a linked list using the blind node short address. After the blasts, the blind node sends a LQI request to all the reference nodes. The reference nodes calculate the average received LQI from the blind node making the request. The blind node waits for $100 \mathrm{~ms}$ so the reference nodes can preform the calculation and send the LQI response back to the blind node. In this response, the reference nodes send the average LQI, the zone and the address of its corresponding location dongle. Once the response has been sent, the reference node deletes the blind node from the linked list. The blind node saves the responses by using the location dongle short address. Under optimized conditions, the blind node receives 4 responses for every location dongle. From these responses, the blind node determines the best LQI and determines the zone number and gets the location dongle's short address. A blind node response to the location dongle with its corresponding zone is sent. The location dongle sends the information to the PC, so it can be processed and displayed. The location dongle sends a message back to the blind node so he can give an indication to the cyclist (turning on a LED) that he has

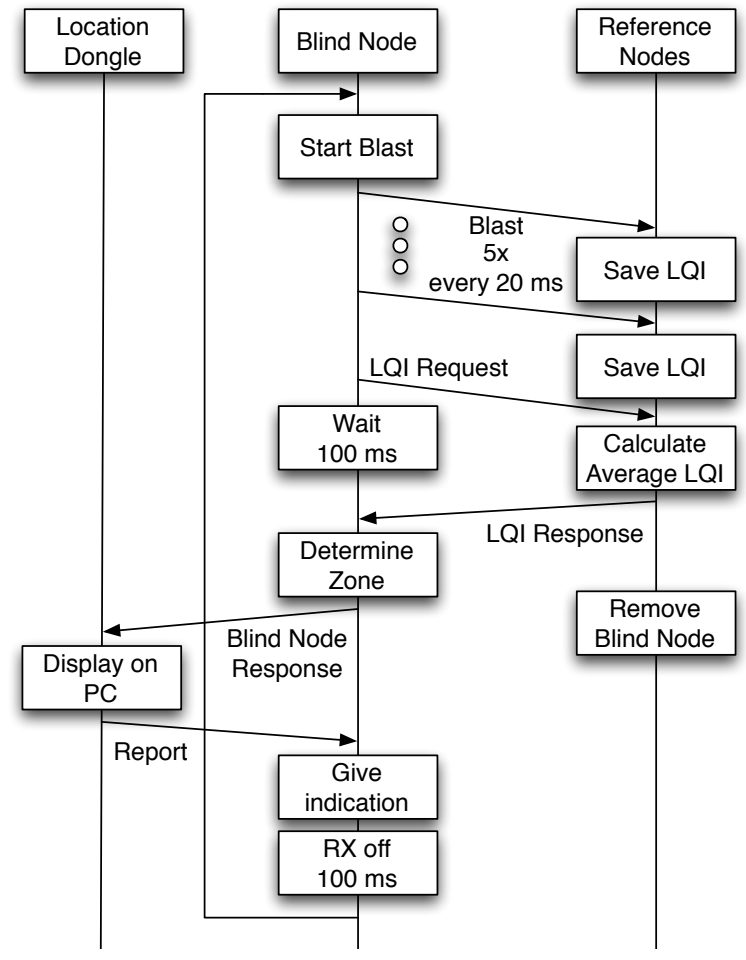

Fig. 3. The data messages between the different nodes

been noticed. The blind node now puts its receiver off for $100 \mathrm{~ms}$ to save power. Before the start of a new blast, the array of location dongles and reference nodes is cleared. This process is repeated as long as the blind node is in the network.

Adding the time that the blind node is in a wait condition, results in $300 \mathrm{~ms}$ (excluding a very small calculation time). This way, the requirement of the update speed is obtained (Table I). There is a possibility to reduce the wait time by sending less blasts or by switching off the receiver for a shorter time. The disadvantage is that the blind node will consume more energy.

If the blind node loses connection with the network because it is out of range and there is no more danger for the cyclist, it will immediately search for a new network that it can join.

\section{Processing by $P C$}

The location dongle sends the messages it has received from the blind nodes to the PC using a serial connection (the blind nodes short address and the zone he has determined). The program makes a linked list of the blind nodes. If the blind node is new, it is added to the linked list by its short address and the parameter "Number of cyclists" on the screen (Figure 4) is immediately incremented. Hereafter, the zone is evaluated and displayed on the screen. The zone is also saved in the linked list. This allows to compare the original position with the new position the blind node sends. At last, 


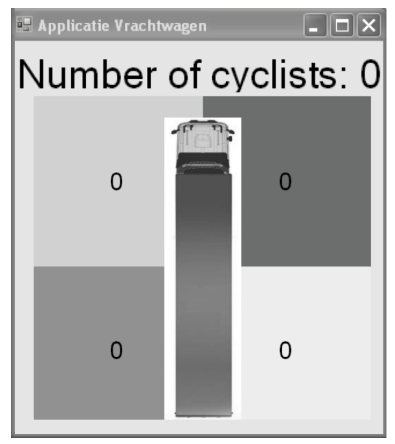

Fig. 4. Illustration of the program on PC

there is also a parameter "Received Message" that is set to 5 . This parameter is used to determine whether the blind node is still in the network or not.

After the blind node is added to the linked list, it sends updates of its zone. The program looks up the blind node by its short address and set the parameter "Received Message" back to 5. Hereafter the program compares the original zone with the update sent by the blind node. When they are equal, no action is taken. When they are different, the zones on the screen are updated immediately. This way the zone is updated at approximately $300 \mathrm{~ms}$ (See Section III-C).

To determine if the blind node has left the network, a periodic timer and the parameter "Received Message" is used. As mentioned above, the parameter "Received Message" is set to 5 every time a message is received from the blind node. Now every $500 \mathrm{~ms}$ the periodic timer passes through the linked list and decrements the parameter "Received Message". If the parameter reaches zero, the blind node has not send a zone update in $2.5 \mathrm{~s}$. In this case, we consider the cyclist to be at a save distance and can therefor be removed from the linked list and the screen. This way a safety margin is included. The blind node must be out of the network for $2.5 \mathrm{~s}$ before it is deleted. If we want to increase the security level, the parameter "Received Message" has to be raised.

\section{TEST RESULTS}

\section{A. Range test}

This first test was meant to determine the range where the blind node loses connection and how the LQI changes according to the distance. For the range test, the LQI is measured from the link between two nodes, which have a LOS connection. The one node is placed at a fixed position, the position of the other node changed from $1 \mathrm{~m}$ to $100 \mathrm{~m}$ in steps of $1 \mathrm{~m}$. Every meter the LQI is measured and the average LQI is calculated over 25 packets. Figure 5 shows the LQI results versus distance. From the test results, a range is determined of approximately $90 \mathrm{~m}$. This is much more than the required range in Table I. The LQI decreases for increasing distances for the first $25 \mathrm{~m}$. After this distance,

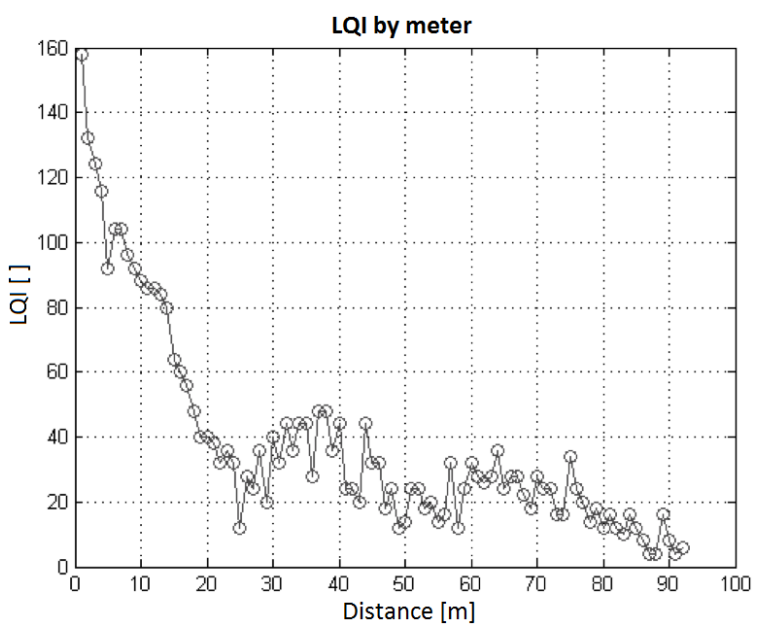

Fig. 5. Test results of the range test

the LQI decreases but a lot of fluctuations occur (Figure 5). It is not necessary that the blind node is placed in a zone at distances greater then $25 \mathrm{~m}$.

\section{B. Detection of the cyclist}

The most important feature of the test setup, is noticing the cyclist. First a static test is done at the right side of a van. Figure 6 shows the configuration for this test. The four reference nodes are placed at the wheels of the van and the location dongle is placed in front of the van. The blind node is placed at a distance of $1.5 \mathrm{~m}$ from the right side of the van. In total there were 14 measuring points. The test time was 5 minutes per measuring point and during that time the parameter "Number of cyclists" and the incoming messages from the blind node were saved in a text file.

For all the measuring points, the parameter "Number of cyclists" on the screen always showed the blind node. The cyclist is always noticed with this static test. But a cyclist moves at speed of approximately $5 \mathrm{~m} / \mathrm{s}$ so also a dynamic test is necessary.

For the dynamic test, a cyclist was moving around the van in a rectangle of $24 \mathrm{~m}$ by $48 \mathrm{~m}$. The van was place in the middle of the rectangle with the same configuration as in the static test. The blind node is mounted on the handlebar of the bike. The cyclist traveled around the van counterclockwise for 5 minutes. This test was repeated 5 times and in all the tests the cyclist was always noticed. The requirement of being noticed is also obtained for the dynamic test.

\section{Placing the cyclist in zones}

The same setup is used with the van and the 14 measuring points of the blind node (Figure 6). The measuring time for every point was 5 minutes. By saving the received messages from the blind node, it is possible to determine if it was 


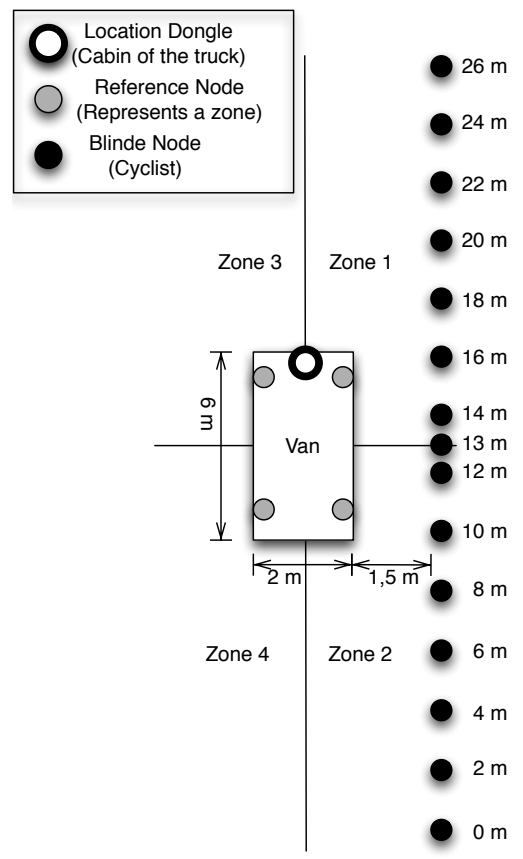

Fig. 6. Configuration of the test

TABLE II

THE PERCENTAGE OF DISPLAYING THE CYCLIST IN THE WRONG ZONE

\begin{tabular}{ll}
\hline Measurement point $(\mathrm{m})$ & Total Error $(\%)$ \\
\hline 0 & 0,41 \\
2 & 0,18 \\
4 & 0,00 \\
6 & 0,64 \\
8 & 0,17 \\
10 & 0,36 \\
12 & 0,39 \\
13 & 0,00 \\
14 & 0,10 \\
16 & 0,00 \\
18 & 0,20 \\
20 & 0,41 \\
22 & 0,20 \\
24 & 0,20 \\
26 & 0,10 \\
\hline
\end{tabular}

placed in the correct zone. Behind the van, the blind node was in zone 2 and by moving to the front, the zone changed to zone 1 . The percentage of placing the cyclist in a wrong zone for this test is shown in Table II for every measuring point. The average error for al the measurement points was only $0,24 \%$ with a maximum of $0,64 \%$.

\section{CONClusion}

In this paper, it is demonstrated that a wireless communication system can be used to suppress the blind spot of a truck. Using Zigbee, a test setup is developed that can be used in a real environment with several trucks and cyclists. From the static and dynamic test results, there can be concluded that the cyclist is always noticed. The requirement of $0,01 \%$ has been obtained. The advantage of the solution with a wireless communication system, is that the truck driver is informed of the cyclist without any personal action. When the cyclist is noticed by the truck driver a signal is given to the cyclist. This way both the truck driver and the cyclist are informed of each other's presence. By our knowledge, there is no solution at the market that informs both participants.

The cyclist is noticed from a very large distance so the truck driver is informed in time of the approach of the cyclist. Most systems on the market only detect cyclists that are in the close neighborhood of the truck (a few meters). When using a wireless communication system, the truck driver can take action before the cyclist is in a dangerous position.

The disadvantage of this Zigbee solution, is the error on determining the correct zone. Maybe, it is possible to use different antennas with a specific opening angle to reduce this error. Another requirement that needs to be tested, is the number of cyclists that can be noticed at the same time. A problem that can occur is a large amount of network traffic so the position update becomes larger than $300 \mathrm{~ms}$.

With this test setup, we have solved a number of issues that are important when a wireless communication system is used to solve the blind spot problem. For example, the most imported issue that has been solved, is the dynamic allocation of reference node. This way a trucker is not bounded to his trailer.

\section{ACKNOWLEDGMENT}

Wout Joseph is a Post-Doctoral Fellow of the FWO-V (Research Foundation - Flanders). We would like to thank all the people who helped and supported this project.

\section{REFERENCES}

[1] Dobli, Blind spot mirror, WWW, http://www.dobli.com/.

[2] Jabor, Blind spot camera, WWW, http://jabor.nl/td/cameras.html.

[3] Blicor, An indication for the cyclists, WWW, http://www.decawave.com.

[4] Life Saver, Lisa2alert blind spot system, WWW, http://www.lisa2alert.com/en/lifesaver.htm.

[5] Lexguard, A sensor based solution, WWW, http://www.lexguard.nl/.

[6] Alan Bensky, Wireless Positioning Technologies and Applications by Alan Bensky, Number 978-1-59693-130-5, Artech House, 2008.

[7] Bill R. and Cap C. and Kafahl M. and Mundt T., Indoor and outdoor positioning in mobile environment - a review and some investigations on wlan positioning, Technical report, University Rostock, Germany, 2004.

[8] Marko Modsching and Ronny Kramer and Klaus ten Hagen, Field trial on GPS Accuracy in a medium size city: The influence of built-up, University of Applied Sciences Zittau/Grlitz, Germany, 2006.

[9] Sensor, Decawave, WWW, http://www.decawave.com/.

[10] Zigbee, Zigbee Alliance, WWW, http://www.zigbee.org/.

[11] Stefano Tennina and Marco Di Renzo and Fabio Graziosi, Locating zigbee nodes using the tis CC2431 location engine: A testbed platform and new solutions for positioning estimation of WSNS in dynamic indoor environments., Technical report, Tunato Santucc.

[12] Texas Instruments, Locate Your Zigbee Solution, WWW, http://www.ti.com/corp/docs/landing/cc2431/index.htm. 